\title{
Contribution of Branch Stories to The Structure of The Epic: A Study Based on The Silappathikaram
}

காப்பியக் கட்டமைப்பில் கிளைக்கதைகளின் பங்களிப்பு : சிலப்பதிகாரத்தினை அடிப்படையாகக் கொண்ட ஆய்வு

Murukaiya Sathees a, *

a Department of Tamil, University of Jaffna, Sri Lanka.

*Corresponding author Email: satheesmurukaiya@gmail.com

DOI: https://doi.org/10.34256/ijmrd2141

Received: 06-09-2021; Revised: 01-10-2021; Accepted: 03-10-2021 Published: 06-10-2021

Abstract: Epic is the Tamil form of the word epic. It is a literary form structured with various elements. Among them the branch is the main component. It is created to support the purpose of coffee. This is the storyline that lies within the central story. Silappathikaram also presents several branch stories with them as a consensual literary form. The central story is the life struggle story of Kovalan - Kannaki - Madhavi, the pioneer of Tamil coffee, Ilangovadikal and Silappathikaram. Several episodes interspersed with the Silappathikara central story expand the scope of the epic story. In particular, 32 episodes co-exist with Kovalan Kannaki's biography, giving a twist, emphasis, clarity and significance to the elemental. These stories complement the silappathikaram storytelling and copy structure. Anecdotes are used in the silappathikaram to reinforce the author's ideas, to advance the narrative and the personality of the characters, and to identify the context and silappathikaram of the narrative and its use. The study focuses on scholasticism with a view to revealing and documenting the role of branch stories in such copywriting. The scholastic epic of the juveniles has been used primarily for this study and related essays, journals, and electronic commentary have also been used as research data. The study also emphasizes that many more such studies should be carried out by researchers in view of the importance of branch stories and their contribution to copywriting.

Keywords: Epic, Silappathikaram, Structure, Anthology, Contribution

Language: Tamil

முன்னுரை

காப்பியம் ஆங்கிலத்தில் 'EPIC' எனப்படுகின்றது. இச்சொல் 'EPOS' என்ற கிரேக்கச் சொல்லில் இருந்து வந்ததாகும். காவியம் என்ற வடசொல்லின் தமிழ் வடிவமே காப்பியம். இது கவியினால் செய்யப்பட்டது என்பர். அத்தகைய காப்பியம் பல்வேறு கூறுகளைக் கொண்டு கட்டமைக்கப்பட்ட இலக்கிய வடிவமாகும். அவற்றுள் கிளைக்கதை முக்கிய கூறாகும். இது காப்பிய நோக்கத்திற்குத் துணைபுரியும் கூறாகப் படைக்கப்படுகின்றது. கிளைக்கதைகள் கூற்றுமுறையில், விபரிப்புப் போக்கில் அமைந்திருக்கும். இத்தகைய கிளைக் கதைகளின் கையாட்சி சிலப்பதிகாரக் காப்பியக் கட்டமைப்பில் எவ்விதம் கையாளப்பட்டுள்ளன என்பதை ஆராய்வதாக இவ்வாய்வு அமைகின்றது. 
ஆய்வு நோக்கம்

காப்பியக்கட்டமைப்பில் கிளைக்கதைகளின் வகிபங்கு எத்தகையது என்பத வெளிப்படுத்தி அவற்றினை ஆவணப்படுத்தும் நோக்கில் சிலப்பதிகாரத்தினை மையப்படுத்தி இவ்வாய்வு மேற்கொள்ளப்பட்டுள்ளது.

\section{ஆய்வு முறையியல்}

இவ்வாய்வானது பண்புசார் முறை மற்றும் விபரணப் பகுப்பாய்வு முறை ஆகிய அணுகுமுறைகளைப் பயன்படுத்தி மேற்கொள்ளப்பட்டுள்ளது. இவ்வாய்விற்கு

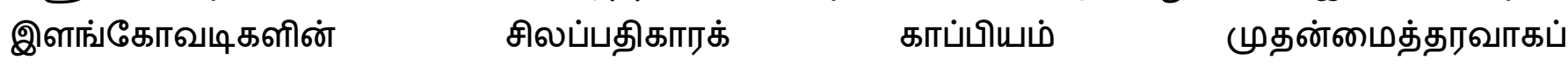
பயன்படுத்தப்பட்டுள்ளதுடன் அது சார்ந்த கட்டுரை நூல்கள், இதழ்கள், மின்னூடகக் கருத்துக்கள் பான்றவையும் ஆய்வுத்தரவுகளாகப் பயன்படுத்தப்பட்டுள்ளன.

\section{காப்பியப்படைப்பில் கிளைக்கதை}

காப்பியங்கள் பல கிளைக்கதைகளைத் தம்முடன் இணைத்து ஒருமித்த இலக்கிய வடிவங்களாகக் காட்சி தருகின்றன. இவ்வாறான காப்பியக்கதையை மூன்று வகைப்படுத்தலாம். மையக்கதை, கிளைக்கதை, துணைக்கதை என்பவையே அவையாகும். இவற்றுள் கிளைக்கதை என்பது மையக்கதையினூடே அமைந்திருக்கும் கதைவடிவமாகும். கிளைக்கதைகள் கூற்று முறையில், விபரிப்புப் போக்கில் அமைந்திருக்கும். மையக்கதை கூறப்படும் அந்தக்காலத்திலன்றி அதற்கு முன் நடந்ததை அல்லது பின்பு நடக்கப்போவதைக் கதைப்படுத்திக் கிளைக்கதைகள் கூறப்படும். மையக்கதையில் இடம்பெறும் பாத்திரங்கள் வாயிலாகவோ, காப்பியப் புலவர் தாமே கூறுவதாகவோ கிளைக்கதைகள் அமைந்திருக்கும். மையக்கதைப்போக்கில் இருந்து வாசகனின் மனதை இக்காப்பியக் களைக்கதைள் திசைதிருப்புகின்றன.

இக்கிளைக்கதை பற்றிய சிந்தனை பழங்காலத்திலிருந்தே ஐரோப்பிய, வடமொழி அறிஞர்களுக்கு இருந்திருக்கிறது. காப்பிய இலக்கணம் பேசும் முதல் நூலான தண்டியலங்காரத்திலோ, பின்வந்த பாட்டியல் நூல்களிலோ கிளைக்கதை பற்றிய செய்தி எதுவுமில்லை. ஆனால் சிலப்பதிகாரத்திற்கு உரை எழுதிய அடியார்க்குநல்லாரிடம் கிளைக்கதை பற்றிய சிந்தனை ஓரளவு இருந்துள்ளமையை அவரது உரையிலிருந்து அறியமுடிகின்றது. ஆனால் அவர் கிளைக்கதைகள் பற்றி விரிவாகப் பேசவில்லை. கிரேக்க அரசியல் ஞானியான 'அரிஸ்டாட்டில்' முதன்முதலாகக் கிளைக்கதை பற்றிய சிந்தனையைப் பழம் காலத்திலேயே தோற்றுவித்தார். அவரே கிளைக்கதையைப் பற்றிய இலக்கணத்தையும் தந்து விளக்கி உள்ளார். கிளைக்கதையை 'EPISODE' என்ற கிரேக்கச் சொல்லால் குறித்தார். பின்வந்த ஐரோப்பிய இலக்கிய அறிஞர்கள் அனைவரும் இச்சொல்லையே கிளைக்கதையைக் குறிக்கப் பயன்படுத்தினர்.

அரிஸ்டாட்டில் கிளைக்கதை பற்றிக் கூறும்போது ‘கிளைக்கதை என்பது காப்பிய மையக்கதையுடன் இணைந்த ஒன்றிய கதையாக வரவேண்டும். காப்பியக் கட்டமைப்புச் செறிவாக இருக்கவேண்டுமானால் கிளைக்கதை மையக்கதையுடன் திறம்பட இணைக்கப்பட்டிருக்க வேண்டும் என்கிறார். இவருக்குப் பின் காப்பிய இலக்கணத்தைப் பற்றி ஆராய்ந்த ப்ளேட்டோ, ஆபர்கிராம்பி, சி.எம்.பௌரா, எப்.எல்.லூகாஸ், கெர் முதலிய இலக்கிய 
அறிஞர்கள் கிளைக்கதையைக் காப்பிய உறுப்பாகச் சுட்டிச் சென்றிருக்கின்றனர். (முத்துச்சண்முகன்.இரா.பிரேமா, இரட்டைக்காப்பியக் கிளைக்கதைகளும் துணைக்கதைகளும், ப.3). அதன் பின்வந்த அகராதிகளும், கலைக்களஞ்சியங்களும் கிளைக்கதைகளை ஆராய்ந்து அவற்றுக்கான பொது விளக்கங்களையும் நல்கியிருக்கின்றன (Muththuchchanmukan, 1980).

• ஒரு நிகழ்ச்சி அல்லது ஒரு காட்சி எடுத்துரைக்கும் பாங்கில் கூறப்பட்டிருக்கும்.

- மூலக்கதையில் இருந்து இயல்பாகத்தோன்றியும், ஆனால் அதிலிருந்து தனிமைப்படுத்தும் விதமாகவும் அமைந்திருக்கும்.

- கிளைக்கதையானது ஒரு மனித வாழ்க்கையில் நடந்த நிகழ்ச்சியாகவும் அமையலாம்.

- கிளைக்கதை மூலக்கதைப்பின்னலுடன் ஒரு சில நேரங்களில் ஒன்றிவருகிறது.

மேலும், வடமொழி அறிஞர்களிடையே கிளைக்கதை என்பது 'உபாக்கியானம்' என வழங்கப்படுகின்றது. உதாரணமாக, பாரதத்தில் இடம்பெற்றுள்ள நளனுடைய கிளைக்கதை 'நளோபாக்கியானம்' என்றும், குசேலருடைய கதை 'குசேலோபாக்கியானம்' என்றும் குறிப்பிடப்படுகின்றது. தமிழ்க்காப்பியங்கள் பற்றி ஆராய்ந்த வையாபுரிப்பிள்ளை, ‘காவியகாலம்' என்ற நூலில் 'கிளைக்கதை பிரதான கதையோடு இயைபு உடையதாய் நாயகன், நாயகி முதலிய முக்கிய பாத்திரங்களின் வரலாறுகளோடு ஏதாவது ஒரு வகையில் தொடர்பு கொண்டதாக அமைய வேண்டும்' என்கிறார்.

\section{கிளைக்கதைகளின் இலக்கணம்}

கிளைக்கதை, தொடர்ந்து விளக்க நடையில் (Descriptive) சொல்லப்படும் காப்பிய மையக்கதையினூடே அமையும் ஒரு கதைநிகழ்ச்சி ஆகும். அதில் ஒரு நிகழ்ச்சி அல்லது காட்சி கதைப்படுத்தப்பட்டிருக்கும். அ அது வெளிப்படையாகவே பரிணமித்துத் தோன்றும். கதையானது படிப்பவரின் ஊகத்திற்கு விடப்படாது. காப்பியப் புலவராலேயே விபரிக்கப்பட்ட தன்மையாய் அமைந்திருக்கும். பெரும்பாலும் கூற்று முறையல் அமைந்திருக்கும். மிகச்சிறுபான்மையாக மாறுபட்டும் அமையலாம். கூற்றுக்குரியவராகக் காப்பியப் பாத்திரங்களோ, காவியப்பாத்திரமல்லாத தெய்வீகப்பாத்திரங்களோ, காப்பியப் புலவரோ அமைவர். காப்பியக் கிளைக்கதைகள் நடந்த கதையாகவே அமைந்திருக்கும். பெரும்பாலும் காப்பியம் நடந்த காலத்திற்கு முன்பு நடந்ததாக அமைந்திருக்கும். சிறுபான்மை இடங்களில் காப்பியம் நடந்த காலத்தில் நடந்திருந்தாலும், நடக்கின்ற நிகழ்ச்சியாக அல்லாமல் நடந்த

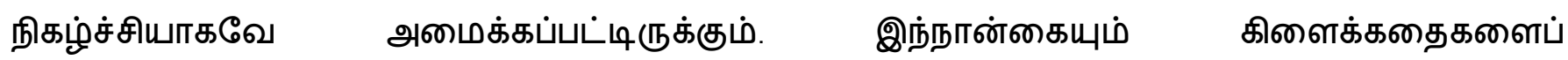
பிரித்தறிவதற்குப் பயன்படும் அடிப்படை இலக்கணங்களாகக் கொள்வர்.

\section{கிளைக்கதைகளாகப் பயன்படுத்தப்படும் கதைகள்}

கிளைக்கதை என்பது புராணக்கதை, புராண வரலாற்றுக்கதை, முன்னோர் கதை, வரலாற்றுவழிக் கதை, கர்ணபரம்பரைக் கதை, பழம்பிறப்புக் கதை, சாப வரலாற்றுக்கதை, முனிவர், அரக்கர், தேவர் பற்றிய கதைகள், பஞ்சதந்திரக் கதைகள், புத்த ஜாதகக் கதைகள் போன்ற கதைகளில் ஓன்றாக அமைந்திருக்கலாம். ஆனால் இக்கதைகள் எல்லா இடங்களிலுமே கிளைக்கதைகளாகவே எடுத்தாளப்படுகின்றன என்று கூறமுடியாது. பல 
இடங்களில் காப்பிய உவமைகளாக எடுத்தாளப்பட்டிருக்கின்றன. சில இ இடங்களில் வர்ணிக்கும் முகமாக எடுத்தாளப்பட்டுள்ளன.

\section{கிளைக்கதைகளின் பயன்பாடு}

கிளைக்கதை மூலக்கதையோட்டத்தின் நிலைப்போக்கை மாற்றியமைக்கின்றது. காப்பியப் பரப்பின் கதையினை விரிவுபடுத்துகின்றது. காப்பியப்பரப்பின் பல்வேறு சூழல்களுக்கும், பண்புகளுக்கும் இடமளிக்கின்றது. கதைப்பின்னலின் முக்கியமான விளைவுகளைச் சுட்டிக்காட்டுகின்றது. கதை நிகழ்ச்சியோடு தொடர்புடைய பிறிதொரு நிகழ்ச்சியை நோக்கி விரியும் போக்கு மற்றும் வாசகர் மனதில் சோர்வைப் போக்கி புத்துணர்வை ஊட்டுவதற்குக் கிளைக்கதை உருவாக்கப்படுகின்றது. சிறிய இடைவெளிக்குப் பின் மையக்கதையை விபரிக்கும் போது படிப்பவரது சுவையுணர்வு தூண்டப்பட்டு காப்பியத்தால் இன்பம் அடைவர்.

\section{சிலப்பதிகாரக் காப்பியக் கட்டமைப்பில் கிளைக்கதைகள்}

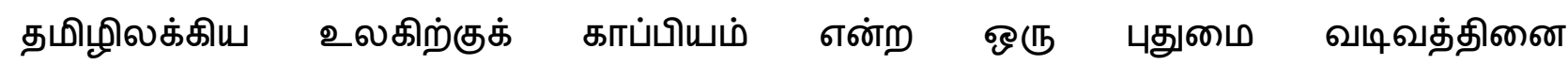
அறிமுகப்படுத்தியவர் இளங்கோவடிகள். சிலப்பதிகாரம் என்ற பெருங்காப்பியத்தைப் படைத்துள்ள இளங்கோவடிகள் புதுமையை விரும்பக் கூடியவர். முற்போக்கான எண்ணம் கொண்டவர். எனவே தான் அவரால் வடிவத்தால், கதைத் தலைப்பால், கதையின் உட்பொருளால் மரபை மீறிய ஒரு மாறுபட்ட இலக்கியத்தைப் படைத்துத் தர முடிந்தது. சிலப்பதிகாரம் மூன்று காண்டங்களையும் முப்பது காதைகளையும் கொண்டது. அவ்விலக்கியத்தின் படைப்புத்திறன் காரணமாக அது இ இன்றும் தமிழ் மக்களிடம் உயிரோட்டம் மிக்க படைப்பாக வாழ்ந்து கொண்டிருக்கின்றது. இக்காப்பியம் 'குடிமக்கள் காப்பியம்' என்றும் 'புரட்சிக் காப்பியம்' என்றும் கூறப்படும் (Subramanian, 2003).

தமிழ்க் காப்பிய முன்னோடியான இளங்கோவடிகள் சிலப்பதிகாரத்தில் கோவலன் கண்ணகி - மாதவி ஆகியோரின் வாழ்க்கைப் போராட்டக்கதையை மையக் கதையாக அமைத்துள்ளார். கண்ணகி - கோவலன் திருமண வாழ்வில் தொடங்கும் மையக்கதை, கண்ணக தெய்வமாகக் கொண்டாடப்படுவதுடன் முடிவடைகின்றது. இம்மையக்கதை கோவலன், கண்ணகி, மாதவியைத் தலைமை மாந்தர்களாகவும் தேவந்தி, கவுந்தி, மாடலன் பாண்டியன், பொற்கொல்லன,; செங்குட்டுவன் ஆகியோரைத் துணை மாந்தர்களாகவும், மாங்காட்டு மறையோன், மாதரி, ஐயைவேண்மாள், கோப்பெருந்தேவி போன்றவர்களைச் சிறு பாத்திரங்களாகவும் கொண்டு திகழ்கின்றது.

ஊழ்வினைப் பயனை மையமாகக் கொண்டு படைக்கப்பட்ட இக்காப்பியத்தில் மாறுபட்ட பல கதை வழங்கப்பட்டு வருவதும் குறிப்பிடத்தக்கவையாகும். இதனைப் பற்றிக் குறிப்பிடும் வி.சி. கந்தையா, கன்னட நாட்டில் வழங்கப்பெறும் சிலப்பதிகாரம் இறுதியில் வெட்டுண்ட கோவலன், கண்ணகியின் கற்பினுக்கிரங்கிய சிவனருளால் உயிர் பெற்றெழ அப்பால் இருவரும் தம்மூர் சென்று சுகமே வாழ்ந்தனரென்று திரிபு கொண்டு வழங்குவதாகக் குறிப்பிட்டுள்ளார். இத்தகைய சிலப்பதிகார மையக்கடலில் கலக்கும் சிற்றாறுகள் எனப் பல கிளைக்கதைகள் இடையிடையே சேர்ந்து காப்பியக் கதையின் பரப்பினை விரிவுபடுத்துகின்றன. குறிப்பாக 32 கிளைக்கதைகள் கோவலன் கண்ணகியின் வாழ்வியல் 
கதையில் இணைந்து நின்று மூலக்கதைக்குத் திருப்பத்தினையும், அழுத்தத்தினையும், தெளிவையும், சிறப்பையும் தந்து நிற்கின்றன. இக்கதைகள் சிலப்பதிகாரக் கதையமைப்பிற்கும் காப்பியக் கட்டமைப்பிற்கும் துணை செய்கின்றன. இக்காப்பியத்தில் ஆசிரியரின் கருத்துக்களை வலுப்படுத்தும் விதத்திலும், கதைத்தன்மையையும், பாத்திரங்களின் ஆளுமையையும் முன்னெடுத்துச் செல்லும் விதத்திலும், கதைச்சூழல், அதன் பயன்பாடு பற்றியதான நிலைப்பாட்டினையும், குறியீட்டுத் தன்மையையும் அடையாளப்படுத்துவதாகக் கிளைக்கதைகள் பயன்படுத்தப்பட்டுள்ளன.

\section{கதைச்சூழலும், கிளைக்கதைச் சுருக்கமும்}

மதுரைக்காண்டம் கட்டுரைக் காதையில் மதுரையை எரித்த பின் உஷ்ணம் தணியாமல் சுற்றித்திரிந்த கண்ணகியின் முன் தோன்றிய மதுராபதி என்ற காவல் தெய்வத்தின் வாயிலாகச் சில கிளைக்கதைகள் முன்வைக்கப்படுகின்றன. அவை கண்ணகியைச் சாந்தப்படுத்தும் நிலையிலும், பாண்டியன் குற்றமற்றவன் என்பதைக் கண்ணகிக்கு உணர்த்தும் வகையிலும் கூறப்படுகின்றன. முன்பொரு சமயம் கணவன் தொழில் வயப்பிரிந்தான். அப்போது ஒரு இரவில் கீரந்தையின் மனைவி, வீட்டல் இருந்தாள். அந்நேரம் நகர காவல் மேற்கொண்ட பாண்டியன் கதவைத் தட்டினான். உள்ளிருந்த கீரந்தையின் மனைவி,

"அரசவேலி அல்லது யாவதும்

புரைதீர் வேலி இல் என மொழிந்து

மன்றத்து இருத்திச் சென்றீர் அவ்வழி

இன்றவ் வேலி காவாதோ?....." (23: 44 - 47) எனக் கூறக்கேட்ட பாண்டிய மன்னன் அச் சொல்லின் வலி தாங்காது தன் கரங்களைத் தானே வெட்டிக் கொண்டான் என அக்கதை அமைகின்றது.

மேலும் கண்ணகி சோழநாட்டைச் சேர்ந்தவள் என்பதால் அவள், நாட்டின் பெருமையைக் கூறி அவளைத் திருப்திப்படுத்தும் எண்ணத்தில் புறாவின் பொருட்டு தன் தசையைக் கொடுத்த சிபிச்சக்கரவர்த்தியையும், பசுவின் பொருட்டு தன் மகனின் உயிரைப் பலியிட்ட மனு நீதிச் சோழனின் பெருமையையும் நினைவுபடுத்துவதும் உளவியல் ரீதியாக முதன்மைக்குரியதாகும். (23: 55 - 60). புகார் காண்டம் கடலாடுகாதையில், சோலையில் தன் காதலியுடன் இருக்கும் விஞ்சையன் இந்திர விழாவிற்குச் செல்லும் பொருட்டு அந்நகர்ச் சிறப்புக்களை தன் காதலியிடம் அறிமுகப்படுத்துகின்றான். அப்போது இந்திரனின் நகரத்தைச் சோழமன்னன் முசுகுந்தன் பாதுகாத்தபோது அவுணர்கள் இருள்கணை ஒன்றை ஏவினார்கள். இதனால் மன்னனுக்குத் துணையாக இந்திரன் ஓர் பூதத்தை அனுப்பினான். அன்று அந்நகரைக் காத்த பூதம் இன்றுவரை சோழர் குடியில் இருந்து பலிகொள்வதாகக் கூறுவர். (6: 7 - 13).

கனாத்திறம் உரைத்த காதையில் கணவனை இழந்த கண்ணகியி துயரத்தை வலுப்படுத்திக் காட்டும் நோக்குடன் குழந்தை, மாலதி, பாசண்டச் சாத்தான், தேவந்தி என்ற பாத்திரங்களைக் கொண்ட ஓர் விரிவான கதை கூறப்படுகின்றது. இங்கு தேவந்தியை மணம் செய்த பாசண்டச்சாத்தான் தெய்வலை அடைதல் காரமாக தேவந்தி 
பிரிவுக்குட்படுத்தப்படுகிறாள். அதன் மூலம் கணவனை இழந்த பெண்ணின் துயரம் வலியுறுத்தப்படுகின்றதனைக் காணலாம். (9 : 5 - 39).

ஊர்காண் கதையில் கோவலன் கண்ணகிக்குத் துன்பம் இளைத்து விட்டேன் என்று வருந்திய போது கவுந்தியடிகள் வாயிலாக அவன் துயர்நீங்கும் பொருட்டும், அவை வினைப்பயன் சார்ந்தவை என்பதை உணர்த்தும் பொருட்டும் இக்கதை வலியுறுத்தப்படுகின்றது. இங்கு சீதையைப் பிரிந்த இராமனின் கதையும், நற்குணம் பொருந்திய தம்பதியைப் பிரிந்து துன்பப்பட்ட நளன் கதையும் நினைவூட்டப்படுவது முக்கியம்பெறுகிறது. (14 : $46 \quad$ - 56). அடைக்கலக்காதையில் மாதவியின் மகளுக்கு "மணிமேகலை” என்று பெயர்சூட்டப்படும் போது முன்னொருமுறை வணிகனைக் கடலில் இருந்து காப்பாற்றிய மணிமேகலா தெய்வத்தின் கதை கூறப்படுகின்றது. $\quad\left(\begin{array}{lllll}15 & \text { : } 27 & \text { - } & \text { 40 }\end{array}\right.$ இக்கதையின் மணிமேகலை, மாணவியின் துன்பத்தைத் தீர்க்க வந்த குறியீடாகவே அடையாளப்படுத்தப்பட்டுள்ளாள்.

மேலும் கோவலனின் கருணையை விளக்குவதோடு இம்மை மறுமை பற்றிப் பேசும் இளங்கோவடிகள், மறையோன் யானையிடம் இருந்து மீட்கப்பட்டதும், ீீரியைக்கொன்ற பார்ப்பினியைக் கணவனுடன் இணைத்ததுமான கதைகள் கூறப்பட்டுள்ளன. (15 : 47 - 53). அத்துடன் பத்தினிமீது பழிசுமத்திய மகனைக் கொல்ல முயற்சி செய்த பூதத்திடம் தன் உயிரை விட்டு மகனைக் காப்பாற்ற முயன்ற தாய், பற்றிய கதையும் முக்கியமானது.

“.... நரகன் உயிர்க்கு நல்லுயிர் கொண்டு

பரகதியிழக்கும் பண்பீங்கு இல்லை..." (15 : 84 - 85) என்று அம்மகனைக் கொன்ற பூதத்தின் கதையும் சிறப்பிற்குரியதொன்றாக விளங்குகின்றது. கொலைக்காதையில் பொற்கொல்லனின் கருத்தை வலுச்சேர்க்கும் விதத்தில் இளவரசனின் நகை திருடப்பட்ட கதையும், மேலும் வலுச்சேர்க்கும் வகையில் காவலனால் கூறப்படும் மற்றொரு திருட்டுக்கதையும் கூறப்பட்டுள்ளன. (16 : 190 - 203).

மதுரைக்காண்டம் வஞ்சினமாலையில் பூம்புகார் நகரத்தில் பெண்களின் சிறப்பை எடுத்தியம்பும் இளங்கோவடிகள், பத்தினிப்பெண்கள் பற்றிய ஏழு கதைகளைக் கூறுகின்றார். ஓரு பெண் காவிரிக்கரையில் தன் தோழியுடன் மணற்பாவை செய்து விளையாடும் போது, இப்பாவை நின் கணவனாவான் எனக் கூறியதால், அங்கேயே நின்று அப்பாவையைக் கணவனாகக்கருதிக் காத்திருந்தாள் ஒரு பத்தினிப் பெண். (21 : 6 - 10). கரிகால் வளவனின் மகள் ஆதிமந்தி ஆட்டணத்தியைக் கணவனாகக் கொண்டாள்;. ஒரு முறை புனலாடும் போது காவிரி வெள்ளம் அவனை இழுத்துச் சென்றது. கடல் அவனைக் கொண்டு வந்து அவள் முன் நிறுத்திக் காட்ட வைத்தாள் (21 : 11 - 15). மேலும் ஒரு பத்தினிப் பெண் கணவன் பிரிந்த நிலையில் அவன் வரும் மட்டும் கல்லாகி, மீண்டும் அவன் வந்தவுடன் கல்லுருவம் நீங்கினாள்; என்ற கதையும் உள்ளது. இதனை,

“...மணல் மலிபூங்கானல் வருகலன்கள் நேக்கிக்

கணவன் வரக் கல்லுருவம் நீத்தாள்;..." (21 : 16 - 17) என்பதன் மூலம் அறியலாம்.

வழிப்போக்கன் ஒருவன் வன்னிமரத்தடியில் பாம்பு கடித்து இறக்கவும், அவனுடன் வந்த பத்தினி அழுது புலம்ப இறைவன் அருளால் உயிர்பெற்றான். இதனை ஊரில் உள்ளவர்கள் நம்ப மறுத்தபோது வன்னி மரத்தையும், மடைப்பள்ளியையும் தனக்குச் 
சாட்டியாகக் கொண்டுவந்து நிறுத்திய கதையும் முக்கியமானது. (21 : 3 - 5). மேலும் மாற்றாளின் குழந்தை தாய் இல்லாத போது கிணற்றில் விழுந்தது. பழிக்கு அஞ்சிய ஒரு பத்தினிப் பெண் தன் குந்தையையும் கிணற்றில் தள்ளிப் பின் இரண்டு குழந்தைகளையும் எவ்வித கேடுமின்றி மீட்டாள். (21 : 18 - 19). இதுதவிர தன் கணவன் வேற்று நாட்டுக்குச் சென்ற போது தன் கற்பைக் காக்கும் பொருட்டு தன்முகத்தை ஒரு குரங்கு முகமாக்கி, கணவன் திரும்பி வந்தபின் தன் பழைய நிலைக்குத் திரும்பினாள் என்ற கதையும் காணப்படுகின்றது. (21: 20 - 23). மேலும் இளமைப்பருவத்தில் வாக்குக் கொடுத்ததற்காகத் தன் மகளுக்குத் தன் தோழியின் மகனைத் திருமணம் செய்து வைத்தாள். (26 : 25 - 34) என்ற பத்தினிப் பெண் பற்றிய கதையும் வழங்கப்பட்டுள்ளது. எனவே காப்பியக்கட்டமைப்பிற்குச் சிறப்புச்சேர்க்கும் விதத்தில்; ஓர் இலக்கிய உத்தியாகக் கிளைக்கதைகளை இளங்கோவடிகள் கையாண்டு உள்ளார். இக்கிளைக்கதைகள் ஆசிரியர் கூறவந்த கருத்தினை எளிதாக மக்களைச் சென்றடைய ஓர் தொடர்பை ஏற்படுத்திக் கொடுக்கின்ற என்பது குறிப்பிடத்தக்கவையாகும் (Soundarapandian, 1988; Subramanian, 1984).

\section{பயன்பாட்டு நோக்கில் கிளைக்கதை அமைப்பு}

சிலப்பதிகாரத்தில் இடம் பெற்றுள்ள கிளைக்கதைகள் பல்வேறு களங்களையும், பலதரப்பட்ட பயன்பாட்டு நோக்கங்களையும் கொண்டுள்ளதாயினும், அமைப்பியல் நோக்கில் ஆய்வுக்குட்படுத்தும் போது அவற்றைப் பொதுவாகக் கீழ்வரும் தலைப்புக்களுக்குள் ஆராயலாம்.

\section{1. ஊழ்வினை முன்வினைப் பயனை உணர்த்தும் கதைகள்}

இத்தலைப்பின் கீழ் சங்கமன் கொலைக்கதை (23 : 151), சீதையைப் பிரிந்த இராமனின் கதை (14 : 48), காட்டில் தமயந்தியைப் பிரிந்த கதை (14 : 55), வணிகனைக்காத்த மணிமேகலா தெய்வத்தின் கதை (15 : 30), எட்டியும் மனைவியும் தேவலோகம் சென்ற கதை (15 : 155) முதலிய கிளைக்கதைகளைக் காணலாம்.

\section{2. நீதியை நிலைப்படுத்திய மன்னர்களின் கதைகள்}

இத்தலைப்பின் கீழ் புறாவிற்காகத்தன் சதையைக் கொடுத்த சிபிச்சக்கரவர்த்தியின் கதை (20 : 52), பசுவிற்காகத் தன் மகіனக் கொன்ற மனுநீதிசோழனின் கதை (20 : 55), வாத்திகன் முன்பு வீழ்ந்து மன்னிப்புக் கேட்ட பாண்டிய மன்னன் கதை (23 : 122), கீரந்தையின் வீட்டில் இரவு கதவைத் தட்டியதற்காகத் தன் கையைத் துண்டித்த பாண்டிய மன்னன் கதை (23 : 52), முதலிய கிளைக்கதைகள் நீதியின் தன்மையை நிலைப்படுத்தும் பொருட்டுக் காணப்படுகின்றன.

\section{3. முன்னிகழ்வை அல்லது பின்னணியை உணர்த்தும் கதைகள்}

இத்தலைப்பை உணர்த்தும் வகையில் மாதவியின் குலமரபை உணர்த்தும் கதை (6 : 20), மணிமேகலைக்குப் பெயர் சூட்டுவதற்காகப் பின்னணியை உணர்த்தும் கதை (15 : 38) முதலியவை காணப்படுகின்றன. 
4. தானத்தின் சிறப்பை வெளிக்கொணரும் கதைகள்

இத்தலைப்பின் கீழ் பாரதப் போரில் உணவு வழங்கிய உதியன் சேரலாதன் கதை (23 : 62), தானத்தால் இறைவனை அடைந்த எட்டி - மனைவி பற்றிய கதை (15 : 196) முதலிய கிளைக்கதைகளைக் காணலாம்.

5. கோவலனின் சிறப்பை உணர்த்தும் கதைகள்

இத்தலைப்பின் கீழ் கீரியைக் கொன்ற பார்ப்பினிக்கதை (15 : 47), பூதம் கொன்ற மகனின் தாய்க்கதை (15 : 84) முதலியவற்றைக் கூறலாம்.

\section{6. பத்தினிப் பெண்களின் கதைகள்}

இந்தத் தலைப்பின் கீழ் மணல் பாவையைக் கணவனாக ஏற்ற பத்தினிப் பெண் கதை (21: 9),வன்னி மரத்தைச் சாட்சி கூறவைத்த பத்தினிப் பெண் கதை (21 : 3), பெருவெள்ளத்தில் தன் கணவனைக் காப்பாற்றிய ஆதிமந்தி கதை (21 : 11), கணவன் வரும் வரை கல்லுருவம் பெற்ற பத்தினிக் கதை (21 : 16), கிணற்றில் விழுந்த குழந்தையை மீட்ட பத்தினிக்கதை (21 : 18), தன் கற்பைக் காக்கும் பொருட்டு தன் முகத்தைக் குரங்கு வடிவமாக்கிய பெண் கதை $(21$ : 21), கொடுத்த வாக்கின்படி தன் மகளுக்கு ஒரு ஆண் குழந்தையைத் திருமணம் செய்து வைத்த பத்தினிப் பெண்ணின் கதை $(21$ : 26) முதலிய கிளைக்கதைகள் கூறப்பட்டுள்ளன.

\section{7. காவல் தெய்வக் கதைகள்}

இத்தலைப்பின் ீீழ் வார்த்திகன் சிறையிலடைப்புக் கதை (23 : 80), சோழநாட்டைக் காத்த பூதத்தின் கதை (6 :10) முதலிய கதைகள் காணப்படுகின்றன.

\section{8. நம்பிக்கைச் சார்புடைய கதைகள்}

இத்தலைப்பில், மதுரைக்குக் கேடுறும் எனக் கூறும் சோதிடக்கதை (23 : 134), சங்கமன் மனைவியின் சாபக்கதை $\quad(23 \quad$ : $151 \quad$ - 137) கிளைக்கதைகளாகும்.

\section{9. பிறரைத் தன்வயப்படுத்தும் உளவியல் சார்ந்த கதைகள்}

இத்தலைப்பில் இளவரசனின் நகை திருடப்பட்ட கதை (16 : 182) மற்றும் காவலனின் வாளைப் பறித்த திருடனின் கதை (16 : 90) முதலிய கிளைக்கதைகள் கையாளப்பட்டுள்ன. இக்கதைகளில் சில ஒரே அமைப்புக்குள்ளாகியும், படிமமாகவும், குறியீட்டு வடிவமாகவும் கையாளப்பட்டுள்ள தன்மைகளைக் காணலாம்.

சிலப்பதிகாரக் கிளைக்கதைகளின் சில தனி இயல்புகள்

சிலப்பதிகாரத்தில் அமைந்துள்ள கிளைக்கதைகளில் பெரும்பான்மையாகப் பாத்திரக் கூற்றுக்களாகவே அமைந்துள்ளன. 'பொற்கொல்லன் கூறும் அரண்மனைத் திருட்டுப்பற்றிய கதை' சிலம்பில் ஒன்றிய கிளைக்கதையாக அமைந்துள்ளது. குறிப்பாக ஒட்டிய கிளைக் கதைகளும், ஊன்று கிளைக்கதைகளும் அதிகமாகவே உள்ளன. ‘ஊர்வசி சாப வரலாறு’ - 
புராணக்கதையாகவும், 'நாளங்காடி பூதக்கதை'- புராண வரலாற்றுக்கதையாகவும் இடம்பெற்றுள்ளன. சிலப்பதிகாரத்தில் சில கிளைக்கதைகள் மிகத்திறம்பட எடுத்தாளப்பட்டுள்ளன. ஒரு கிளைக்கதை, பின்னால் வரும் மற்றொரு கிளைக்கதைக்கு அழுத்தம் தருவதாக அமைக்கப்பட்டிருக்கும் போக்கை ஏனைய காப்பியங்களில் காண்பதரிது. ஏனைய காப்பியங்களுடன் ஒப்பிடும் போது சிலம்பில் எந்தவொரு கிளைக்கதையும் தனித்துச் சமயம் பேசுவதற்காக எடுத்தாளப்படவில்லை என்பதும் குறிப்பிடத்தக்கது. 'இளங்கோ அரசு துறந்த வரலாறு' என்பதை காப்பியக் கிளைக்கதை மூலம் கூறுவதால், காப்பியப் புலவரும் தன்னைக் காப்பியத்தில் இடம்பெற வைத்துக் கொண்ட தன்மையைக் காணலாம். ஒரே செய்தியை (ஊர்வசி சாப வரலாறு) வர்ணனையாகவும், கிளைக்கதையாகவும் கையாண்டுள்ள தன்மையும் சிலப்பதிகாரத்தில் காணமுடிகின்றது.

முடிவுரை

சிலப்பதிகாரம் பல கிளைக்கதைகளைத் தன்னுடன் இணைத்து, ஒருமித்த இலக்கிய வடிவமாய்த் தான் கூறவந்த மையக்கதையை ஆணித்தரமாய்ப்பதிவு செய்துள்ளது. இலக்கியமானாலும் சரி பிற கலைகளாயினும் சரி ஒரு நிகழ்வை மங்கலமாக முடிவிற்குக் கொண்டு வருவதென்பது இந்திய மனக்கட்டமைப்பு. அந்த நிலைப்பாட்டில் இருந்து முரண்பட்ட தன்மையோடு காப்பியத் தலைவன் கொலை செய்யப்பட்ட நிலையில் கதையினை முடிவுக்குக் கொண்டுவருவது இளங்கோவடிகளின் முதல் வெற்றியாகும். அந்நிலையில் படைக்கப்பட்டாலும் மக்களால் ஏற்றுக்கொள்ளப்பட்டு மக்கள் இலக்கியமாக விளங்குவதற்கு ஏதுவாகக் கிளைக்கதைகள் மையக்கதைக்குப் பக்கபலமாக அமைக்கப்பட்டுள்ளன. கிளைக்கதைகள் இல்லையெனில் காப்பியம் வெறுமையமைந்து விடும். காப்பிய வனப்பை மெருகூட்டவும், வாசக ஆர்வத்தைத் தூண்டவும் அவை பயன்படுகின்றன. இத்தகைய சிறப்புக்களைக் கொண்டு விளங்கும் கிளைக்கதைகளின் பங்களிப்பு என்பது சிலப்பதிகாரத்தில் கணிசமானதாகும். குறிப்பிட்டுச் சொல்வதானால் கிளைக்கதைகளற்ற ஓர் சிலப்பதிகாரம் இலக்கிய உலகில் இந்த அளவிற்கான பாதிப்பை ஏற்படுத்தும் சாத்தியக் கூறுகள் உண்டா? என்பது கேள்விக்குரியதாகவே அமைகின்றது. எனவே காப்பிய மையக்கதையின் போக்கிற்குக் கிளைக்கதைகளின் பங்களிப்பு இன்றியமையாததாகும். கிளைக்கதைகளின் முக்கியத்துவம் காப்பியக்கட்டமைப்பில் பெறும் பங்களிப்பினைக் கருத்திற்கொண்டு இதுபோன்ற மேலும் பல ஆய்வுகள் ஆய்வாளர்களால் மேற்கொள்ளப்பட ேேண்டும் என்பதனையும் இவ்வாய்வு வலியுறுத்துகின்றது.

\section{References}

Muththuchchanmukan. (1980). Double Copy Branches and Supplements. Madurai: Muththu Publishing House.

Soundarapandian, s. (1988). Epics in Tamil. Chennai: Star Publication.

Subramanian, S.W. (1984). Literary Strategies of Ilango. Chennai: World Tamil Research Institute.

Subramanian, V. (2003). Silappathikaram Panpadduchchurangam. Chennai: Manivasakhar Publishing House. 


\section{Funding}

No funding was received for conducting this study.

\section{Conflict of interest}

The Author has no conflicts of interest to declare that they are relevant to the content of this article.

\section{About the License}

(C) The author 2021. The text of this article is open access and licensed under a Creative Commons Attribution 4.0 International License

\section{Cite this Article}

Murukaiya Sathees, Contribution of Branch Stories to The Structure of The Epic: A Study Based on The Silappathikaram, Vol 2, Iss 4 (2021) 1-10. DOl: https://doi.org/10.34256/ijmrd2141 